\title{
Asherman's syndrome after removal of placenta remnants: a serious clinical problem
}

\author{
A. B. Hooker • A. Thurkow
}

Received: 14 March 2011 / Accepted: 28 April 2011 /Published online: 19 May 2011

(C) Springer-Verlag 2011

\begin{abstract}
Intrauterine adhesions (IUA) or Asherman's syndrome is thought to develop after trauma to the uterine cavity by destruction of the basal layer of the endometrium. IUA can result in menstrual disorders, infertility, and complication during pregnancy and delivery. IUA formation is multifactorial, with pregnancy being an important etiologic factor. Performing a postpartum exploration/ evacuation or curettage can lead to adhesion formation. We present three patients who presented with a menstrual disorder after postpartum surgical intervention on suspicion of placental remnants. Hysteroscopic evaluation revealed severe intrauterine adhesions with complete obliteration of the uterine cavity. Repeated and extensive hysteroscopic adhesiolysis is performed to acquire a cavity with a normal appearance. Besides the puerperal uterus, the time of surgical performance is crucial in the risk for adhesion formation. Performing a late surgical intervention, as from $24-48 \mathrm{~h}$ after delivery, leads to an increased risk for adhesion formation. Prevention of IUA can be established by an accurate indication for late postpartum surgical interventions. When performing a late surgical intervention, hysteroscopic surgery is preferable. Firstly, hysteroscopy allows the possibility for identification of placental remnants, and secondly, the possibility for selective removal, thus avoiding unnecessary trauma to the endometrium compared to blindly curettage. Caution is advised when performing a late puerperal surgical intervention. An accurate indication is essential, and when
\end{abstract}

A. B. Hooker $(\triangle) \cdot$ A. Thurkow

Department of Obstetrics and Gynaecology,

Sint Lucas Andreas Hospital,

Amsterdam, The Netherlands

e-mail: a.hooker@slaz.nl needed, hysteroscopic surgery is preferable, minimizing trauma to the endometrium.

Keywords Asherman's syndrome - Adhesion - Pregnancy . Placental remnants · Surgical intervention · Puerperium . Prevention $\cdot$ Curettage

\section{Background}

Intrauterine adhesion (IUA), also known as posttraumatic amenorrhea, was first described in 1894 by Fritsch [1]. In 1948, Joseph Asherman was the first to describe the frequency of this syndrome, which has borne his name ever since [2]. Asherman's syndrome, defined as adhesion in the uterine cavity, is thought to develop following trauma to the uterine cavity by destruction of the basal layer, the regenerative reservoir of the endometrium. In the healing process, fusion between the injured opposing uterine walls may arise, and as a consequence, partial or complete obliteration of the uterine cavity may occur [3, 4]. IUA can cause menstrual disturbances, infertility, and recurrent abortions. If pregnancy occurs, it is frequently complicated by miscarriage, ectopic pregnancy, abnormal placentation, fetal growth restriction, fetal anomalies, premature labor and delivery, and postpartum hemorrhage $[3,5,6]$.

IUA formation is multifactorial with multiple predisposing and causal factors. The specific cause of IUA formation is difficult to determine because the true pathophysiological process that leads to IUA formation is still obscure. Pregnancy appears to be an important etiologic cause as it is one of the dominating predisposing factors in $91 \%$ of the patients with adhesions; adhesions appear in $67 \%$ after miscarriage curettage and in $22 \%$ after postpartum curettage [3]. Other studies have confirmed the predisposing condi- 
tion of the gravid uterus [6-9]. Besides the gravid uterus, other determinants as surgery, infection, inflammation, and constitutional characteristics play a role in adhesion formation. There is a lack of studies/reports addressing the prevalence of adhesion formation, especially in relation to puerperal surgical interventions.

\section{Method}

The indication to perform a secondary postpartum curettage and/or digital evacuation is suspicions of placental remnants. The surgical procedure is often performed in daily practice, but there is lack of information concerning frequency and possible complications. We present three patients who developed severe adhesions and Asherman's syndrome after undergoing surgical treatment on suspicions of placental remnants after delivery. We propose treatment strategies to prevent or reduce the formation of adhesions.

\section{Case 1}

A 34-year-old primigravida was initially in primary care with an independent midwife. At 33 weeks of gestation, the membranes ruptured, and she was transferred to our teaching hospital. She was treated with steroids to enhance pulmonary maturity and calcium channel blocker (nifedipine) for tocolysis. Despite tocolytic therapy, the patient was in labor and spontaneously delivered a healthy girl, weighing 2,000 g (P50) with 1- and 5-min Apgar scores of 9 and 10, respectively. The newborn baby was admitted at the neonatal intensive care unit. The retained placenta was managed by manual removal under general anesthesia. The placenta localized at the right fundus could be easily removed. The uterine cavity was normal, in particular, no signs of congenital uterine anomalies. The estimated amount of blood loss was 2,500 $\mathrm{ml}$, and blood transfusion was not necessary. After a short observation period, the patient and newborn could be discharged in good clinical condition.

Two months later, the patient still had irregular blood loss. Transvaginal ultrasonography was performed to evaluate the blood loss. A white, not well-defined structure in the uterine cavity was detected with a maximum diameter of 5.2 by $2.7 \mathrm{~cm}$ (Fig. 1). The structure had the echogenic appearance of placenta tissue. A puerperal curettage was performed; macroscopic placental tissue was removed. The postoperative period was unremarkable. Histological examination proved placental remnants.

Six months after delivery, the patient was referred because of secondary amenorrhea. An attempt to perform a saline infusion sonography (SIS) failed because of a cervical stenosis. Hysteroscopic adhesiolysis under general anesthesia was performed. Because of the cervical stenosis,

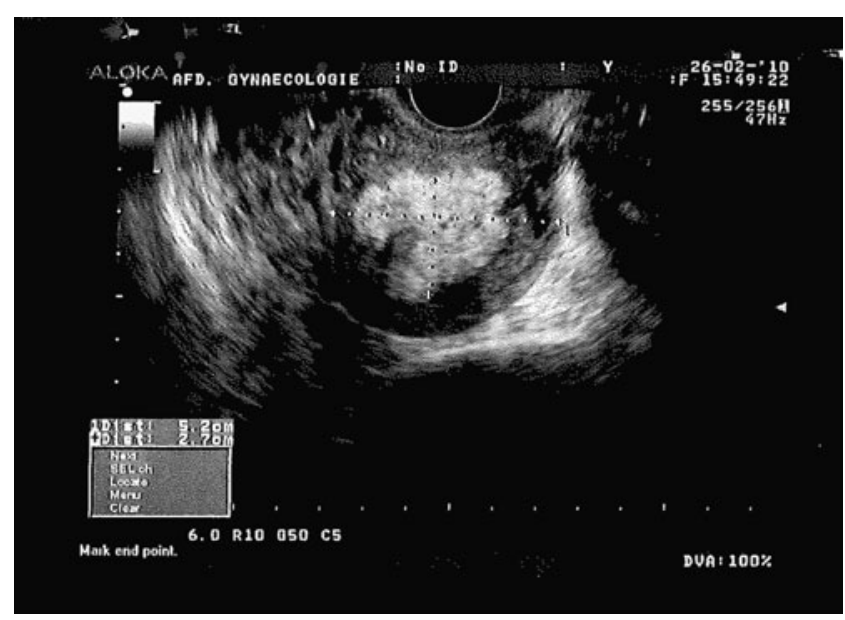

Fig. 1 Transvaginal ultrasonography performed 8 weeks after delivery. A white, not well-defined structure is seen in the uterine cavity, with a maximum diameter of $5.2 \times 2.7 \mathrm{~cm}$. The structure has the echogenic appearance of placenta tissue

introduction was difficult; the uterine cavity was reached but was unrecognizable. A small channel could be detected at the left side connected with the left tube. A hysterosalpingography performed during the operation confirmed the image (Fig. 2). At the right side, a niche was found, probably the remains of the right side of the uterine cavity. The niche could not be reached by hysteroscopy; adhesiol-
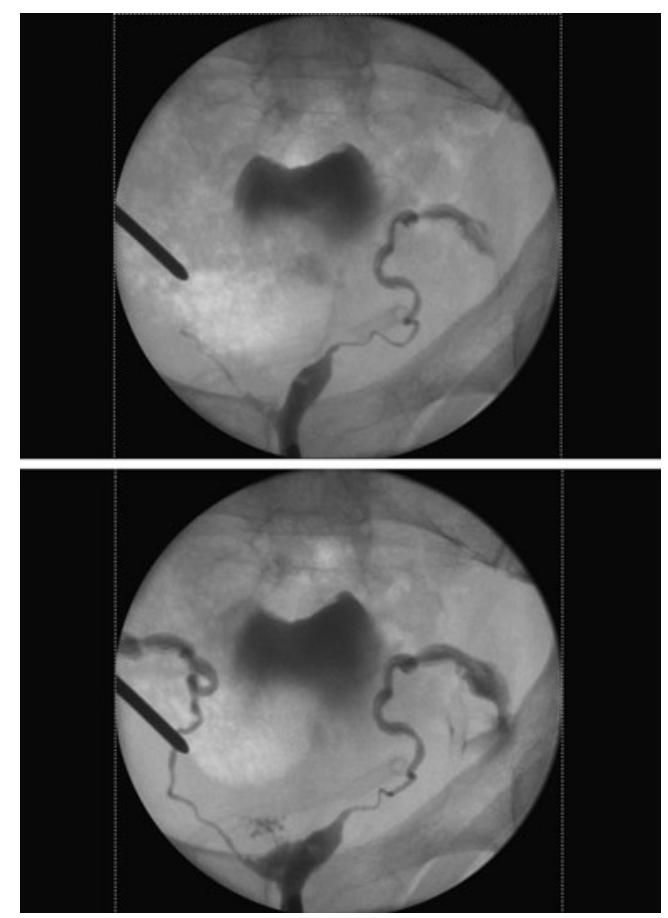

Fig. 2 Hysterosalpingography performed in case 1. Top: At the left side, a small channel is connected with the left tube. At the right side, no cavity can be visualized; there seems to be minimal connection with the right tube. Bottom: After extensive adhesiolysis, a normal uterine cavity with connection with both tubes is visible 
ysis was performed. After adhesiolysis, the cavity was enlarged, but still incomplete. A second session was necessary. After extensive adhesiolysis, the uterine cavity had a normal appearance. After the procedure, the patient regained a normal menstrual cycle. One year later, the patient still has a regular menstrual cycle.

\section{Case 2}

A 34-year-old primigravida with an unremarkable medical history was initially in primary care but was transferred in late pregnancy to our teaching hospital because of pregnancy-induced hypertension. The labor was induced by amniotomy and intravenous administration of oxytocin. The patient spontaneously delivered a healthy girl, weighing 3,500 g with 1- and 5-min Apgar scores of 9 and 10, respectively. Spontaneous placental separation and delivery occurred. Because of a primary postpartum hemorrhage despite treatment with uterotonic drugs, digital exploration and evacuation under general anesthesia was performed. The uterus was atonic and filled with clots. After removal of blood clots, the uterine cavity felt normal; there were no signs of congenital uterine malformations or placental remnants. Contraction of the uterus and normalization of the blood loss occurred after additional treatment with uterotonic drugs. The estimated blood loss was $4,000 \mathrm{ml}$; the patient received 4 units of red cells and 2 units of fresh frozen plasma. After a short clinical observation, the patient was discharged in good clinical condition.

Two months later, the patient reported persistent, irregular blood loss. Ultrasonography showed a uterine cavity with a structure suspicious for placenta remnants. Curettage was performed with removal of tissue. Histological examination did not prove placental residue.

Six months after delivery, the patient was referred because of amenorrhea. The patient stopped lactation for several months; thereafter, no menstrual bleeding occurred. A SIS was attempted, but was inconclusive because of insufficient distension or enlargement of the cavity. An outpatient hysteroscopy showed exhaustive adhesions. A second procedure under general anesthesia was planned. Introduction of the hysteroscope was difficult because of a cervix stenosis; the passageway of the cervix was narrow and fibrotic. Because of the unclear picture, a hysterosalpingography was performed during operation, which shows an asymmetric uterine cavity with a remarkable wide open right tube (Fig. 3). Hysteroscopically, the cavity was reached and was unrecognizable. At the right side, a niche was visible with endometrium, which could be followed into the right, distended tube, possibly a hydrosalpinx. At the left side, no cavity could be identified, and the origin of the left tube is identified by hysterosalpingography; with the hysteroscope, the origin of the left tube was not visible.

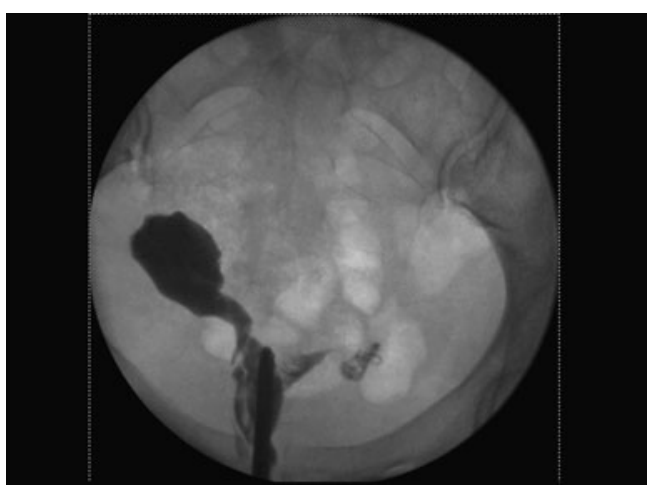

Fig. 3 Hysterosalpingography. At the right side, a niche is visible, which is connected to the distended right tube, possibly a hydrosalpinx. At the left side, no cavity or left tube could be identified, and the origin of the left tube cannot be identified

Adhesiolysis was started, but because there was difficulty in identifying the boundaries of the cavity and suspicion of intra-abdominal involvement, a new combined session with hysteroscopy, hysterosalpingography, and laparoscopy was planned.

At the third session, the uterine cavity could ultimately be reached under transrectal ultrasonographic guidance. The impression of the cavity is equal to that of the second session. With the combination of hysteroscopy and hysterosalpingography, the right tube and the right side of the cavity could be identified. At laparoscopy, the right tube ran into the uterine cavity through a perforation gap near the origin of the right tube. First, laparoscopic adhesiolysis was performed; thereafter, the right tube could be removed from the cavity. The uterine defect was closed after excision of the margins. Secondly, hysteroscopic adhesiolysis was performed with removal of the remains of the fimbriae, ultimately with a more or less normal cavity. For the prevention of adhesions, Hyalobarrier ${ }^{\circledR}$ was administered, but because of persistent blood loss, a Cook balloon was inserted. The patient was treated with an additional regimen of cyclical estrogen and progesterone for 7 days during two cycles. A fourth and last session for hysteroscopic check-up revealed a more or less normal cavity. The patient has a regular cycle.

\section{Case 3}

A 28-year-old gravida 3 para 1, with a spontaneous delivery and a miscarriage in history, was followed in primary care by an independent midwife. At 39 weeks of gestation, she spontaneously delivered a healthy boy, weighing 3,950 g with 1- and 5-min Apgar scores of 9 and 10, respectively. Because of a primary postpartum hemorrhage, the patient was transferred to secondary care. Treatment with uterotonic drugs normalized the blood loss, and the patient was discharged in good clinical condition after a short observation period. 
Three weeks later, the patient was readmitted because of excessive bleeding. Ultrasonographic imaging was performed. Curettage was performed because of suspicion of placental remnants, and macroscopic placental tissue was removed. The postoperative course was uncomplicated. Because of a hemoglobin level of $4.2 \mathrm{mmol} / \mathrm{l}$, iron therapy was started. Histological evaluation proved placental remnants.

Six months later, the patient was referred to our outpatient department because of pelvic pain and amenorrhea, despite the use of oral contraceptive. Transvaginal ultrasonography revealed a thin endometrium, and the image of the uterine cavity was not clear. A SIS could not be performed because the catheter could not be introduced in the cervical channel; cervix stenosis was suspected. A hysteroscopy was performed; an obliterated cervix was seen and could be opened after adhesiolysis under ultrasonographic guidance. After reaching the uterine cavity, widespread adhesions were encountered with an obliterated cavity. Adhesiolysis was performed under ultrasonographic guidance. The ostia of the left tube could be identified, but the right ostia was not visible. A hysterosalpingography performed during surgery confirmed the image. Because of intravasation of contrast medium, proper imaging was limited, and the operation was ended. A second session was planned. At the outpatient department visit, the patient reported that she was menstruating again. The patient canceled the second procedure because of a regular menstrual cycle.

\section{Findings}

Postpartum curettage and/or digital exploration/evacuation are considered the standard surgical therapy when there is the suspicion of placental remnants or hemorrhage. It is unknown how often a secondary surgical procedure is performed and of the possible complications.

The reported cases show that secondary puerperal interventions can lead to serious complications. Performing a postpartum exploration/evacuation or curettage on suspicion of placental remnants can lead to adhesion formation -Asherman's syndrome. Therefore, caution is advised when performing intrauterine interventions in both patients with an earlier intrauterine intervention (cases 1 and 2) as in patients without (case 3). Although only a few studies are available in which the incidence of intrauterine adhesions after secondary puerperal intervention is assessed, they imply a serious clinical problem.

Curettage alone does not seem to predispose to adhesion formation, as in the nonpregnant uterus, intrauterine adhesions post-curettage are reported in $0.4-1.2 \%$ of patients [3]. Pregnancy, however, appears to be a contrib- uting factor, as intrauterine adhesions have been reported in $2 \%$ after manual removal [10]. Also, curettage, exploration, or evacuation performed between 2 and 4 weeks after delivery leads to a substantial increase in adhesion formation varying between $29 \%$ and $37.5 \%$ [11-13]. The timing of surgical intervention also appears to be important as intervention after $24-48 \mathrm{~h}$ post-delivery appears to increase the risk of adhesion formation [12, 13]. Even more concerning and disturbing is that, besides the increase chance of adhesion formation, in $30-60 \%$, the adhesions are of a severe stage $[12,14]$.

IUA formation is considered multifactorial, although the true pathophysiological process is still unknown. The specific characteristics of the puerperal uterus and time of intrauterine intervention are important predisposing factors in adhesion formation. The influence of other factors still remains obscure.

A possible explanation for the enhanced risk and severity of the reported adhesions is the fact that the endometrium of the gravid uterus is in a recovering state. The manipulation disturbs or influences the healing process in an irreparable way, implicating the ultimate result. Destruction of the basal layer of the endometrium, in the healing process probably is one of the key factors in the process leading to adhesion formation.

A second explanation could be the presence of an infection or inflammation. The prolonged presence of placenta tissue in the uterine cavity could enhance infection. Infection is a known situation with enhancement of adhesion formation [4]. In $56 \%$ of the patients after puerperium curettage, performed in the second to fourth week during puerperium, histological examination shows the presence of acute, subacute, or chronic inflammation without symptoms that would suggest endometritis [15]. Low estrogen state, including the puerperium, seems to significantly increase the risk of developing adhesions [12]. Lactation, also being a hypoestrogenic condition, could be an important additional factor in adhesion formation.

Instead of curettage, which is obviously performed blindly, evaluation of the uterine cavity can be conducted by hysteroscopy. Hysteroscopy is considered a reliable technique for identification of intrauterine pathology, including placental remnants [16]. Direct vision of the uterine cavity enables identification of placental remnants. In the absence of placental tissue, intervention and trauma to the endometrium can be prevented.

If placental remnants are present, hysteroscopic removal of placental remnants is a good alternative [16]. Hysteroscopy has the advantage of the placental remnants being evacuated selectively, minimizing trauma to surrounding endometrium and basal layer [17]. Hysteroscopic removal of placenta tissue can easily be performed without increase of complications. Both in the absence as in the presence of 
placental remnants, trauma to the endometrium is reduced compared to curettage. Reduction of trauma to the endometrium reduces the risk of adhesion formation. In view of the benefits, hysteroscopic evaluation should be the treatment of choice whenever a secondary intervention is performed on suspicion of placental remnants [18].

The prevention of intrauterine adhesions is important; adhesions are associated with menstrual and fertility disorders and complications during pregnancy and delivery. In order to minimize the amount of (unnecessary) surgical interventions in the puerperium, it is important to strictly maintain the indication for postpartum curettage and to consider medical evacuation. If a surgical intervention is necessary, preferable hysteroscopically, it should be performed in the gentlest manner, avoiding unnecessary trauma. Application of preventive matters for the prevention or reduction of adhesion can be considered, but only a minority of these products has been studied in women postpartum.

\section{Conclusion}

The gravid uterus is highly predisposed to adhesion formation. Besides the puerperal uterus, the time of postpartum curettage, evaluation, or exploration performance is crucial in the risk for adhesion formation.

When performing an intrauterine surgical intervention after more than 24-48 $\mathrm{h}$, the chance of adhesion development significantly increases. An accurate indication is essential, and when performed, it should be in the gentlest manner, avoiding unnecessary trauma. Hysteroscopic treatment should be the preferred treatment; selective removal of placental remnants is possible with reduced trauma to the uterine cavity compared to curettage. Hysteroscopy minimizes the amount of unnecessary surgical intervention and trauma to the uterine cavity. Further research is necessary to analyze IUA formation and preventive measures.

Declaration of interest The authors report no conflicts of interest. The authors alone are responsible for the content and writing of the paper.

\section{References}

1. Fritsch H (1894) Ein Fall von volligen Schwund der Gebaumutterhohle nach Auskratzung. Zentralbl Gynaekol 18:1337-1342

2. Asherman JG (1948) Amenorrhoea traumatica (atretica). J Obstet Gynaecol Br Emp 55(1):23-30

3. Schenker JG, Margalioth EJ (1982) Intrauterine adhesions: an updated appraisal. Fertil Steril 37(5):593-610

4. Schenker JG (1996) Etiology of and therapeutic approach to synechia uteri. Eur J Obstet Gynecol Reprod Biol 65(1):109-13

5. Capella-Allouc S, Morsad F, Rongières-Bertrand C, Taylor S, Fernandez H (1999) Hysteroscopic treatment of severe Asherman's syndrome and subsequent fertility. Hum Reprod 14 (5): $1230-3$

6. Valle RF, Sciarra JJ (1988) Intrauterine adhesions: hysteroscopic diagnosis, classification, treatment, and reproductive outcome. Am J Obstet Gynecol 158(6):1459-70

7. March CM (1995) Intrauterine adhesions. Obstet Gynecol Clin North Am 22(3):491-505

8. Fernandez H, Al-Najjar F, Chauveaud-Lambling A, Frydman R, Gervaise A (2006) Fertility after treatment of Asherman's syndrome stage 3 and 4. J Minim Invasive Gynecol 13(5):398402

9. Friedler S, Margalioth EJ, Kafka I, Yaffe H (1993) Incidence of post-abortion intra-uterine adhesions evaluated by hysteroscopya prospective study. Hum Reprod 8(3):442-4

10. Golan A, Raziel A, Pansky M, Bukovsky I (1996) Manual removal of the placenta-its role in intrauterine adhesion formation. Int J Fertil Menopausal Stud 41(5):450-1

11. Jensen PA, Stromme WB (1972) Amenorrhea secondary to puerperal curettage (Asherman's syndrome). Am J Obstet Gynecol 113(2):150-7

12. Westendorp IC, Ankum WM, Mol BW, Vonk J (1998) Prevalence of Asherman's syndrome after secondary removal of placental remnants or a repeat curettage for incomplete abortion. Hum Reprod 13(12):3347-50

13. Eriksen J, Kaestek C (1960) The incidence of uterine atresia after post-partum curettage. A follow-up examination of 141 patients. Dan Med Bull 7:50-1

14. Dawood A, Al-Talib A, Tulandi T (2010) Predisposing factors and treatment outcome of different stages of intrauterine adhesions. J Obstet Gynaecol Can 32(8):767-70

15. Smid I, Bedö T (1978) Curettage during puerperium and its late consequences. Zentralbl Gynäkol 100(14):916-20

16. Cohen SB, Kalter-Ferber A, Weisz BS, Zalel Y, Seidman DS, Mashiach S, Lidor AL, Zolti M, Goldenberg M (2001) Hysteroscopy may be the method of choice for management of residual trophoblastic tissue. J Am Assoc Gynecol Laparosc 8(2):199-202

17. Goldenberg M, Schiff E, Achiron R, Lipitz S, Mashiach S (1997) Managing residual trophoblastic tissue. Hysteroscopy for directing curettage. J Reprod Med 42(1):26-8

18. Ventolini G, Zhang M, Gruber J (2004) Hysteroscopy in the evaluation of patients with recurrent pregnancy loss: a cohort study in a primary care population. Surg Endosc 18(12):1782-4 